\title{
Perceptual interference at encoding enhances item-specific encoding and disrupts relational encoding: Evidence from multiple recall tests
}

\author{
NEIL W. MULLIGAN \\ Southern Methodist University, Dallas, Texas
}

\begin{abstract}
Interfering with perception during encoding can enhance later memory, a phenomenon known as the perceptual interference effect. This effect is investigated in the context of the item-specific-relational framework (e.g., Hunt \& McDaniel, 1993), which suggests that the perceptual interference enhances item-specific encoding and impedes relational encoding. Two experiments performed with multiple recall tests support this view. Prior research indicates that item-specific processing increases item gains across tests, whereas relational processing protects against item losses (e.g., Burns, 1993; Klein, Loftus, Kihlstrom, \& Aseron, 1989). Consistent with the item-specific-relational framework, perceptual interference produced significant increases in both item gains and losses relative to a control condition.
\end{abstract}

Interfering with word perception during encoding can enhance later explicit memory, a phenomenon dubbed the perceptual interference effect (e.g., Hirshman \& Mulligan, 1991; Mulligan, 1996; Nairne, 1988). In studies of this effect, words are encoded under one of two conditions. In the perceptual interference condition, each word is presented on a computer screen very briefly (e.g., $100 \mathrm{msec})$ and then backward masked with a row of Xs. In the intact condition, the word is presented unmasked for a longer duration (e.g., $2.5 \mathrm{sec}$ ). In both cases, the subject's task is to identify the word. Subsequently, the subject's memory for the words is tested. Surprisingly, the perceptual interference condition leads to superior performance on a variety of explicit memory tests, including free recall, recognition, and cued recall (see Mulligan, 1999, for a review).

Hirshman, Trembath, and Mulligan (1994) and Mulligan (1996) have tested several potential accounts of the perceptual interference effect (e.g., differential rehearsal, elaborative rehearsal, demand characteristics, encoding effort, visual distinctiveness). Of the accounts considered, only the compensatory processing account was consistent with the complete pattern of results (see Hirshman et al., 1994; Mulligan, 1996, for discussion). The compensatory processing account proposes that the backward mask produces difficulties in the visual processing of perceptual interference items, forcing participants to perform additional higher level (nonvisual) processing. This account is based on studies of perception and word recognition, which have demonstrated that phonological, lexical, and semantic information play a role in word perception (e.g., Balota, 1990; Carr \& Dagenbach, 1990; Seidenberg \& McClel-

I thank Tom Carr, Peter Graf, and an anonymous reviewer for helpful comments on an earlier version of this manuscript. Address correspondence to N.W. Mulligan, Department of Psychology, Southern Methodist University, Dallas, TX 75275-0442 (e-mail: mulligan@mail.smu.edu). land, 1989; Strain, Patterson, \& Seidenberg; 1995). Consistent with formal models of word perception (e.g., Plaut, McClelland, Seidenberg, \& Patterson, 1996; Seidenberg \& McClelland, 1989), the compensatory processing view states that the presentation of the word's visual features begins activation of higher level information (such as phonology, meaning, and/or abstract lexical information). At some point during the course of processing, the mask renders the visual information useless, forcing the perceptual system to rely on the partially activated higher level information. According to the compensatory processing account, the differential processing of this higher level information enhances later memory (see Hirshman et al., 1994, for more complete discussion).

Although counterintuitive, the perceptual interference effect is similar to other encoding effects in which identification of impoverished or transformed stimuli leads to superior memory. For instance, reading inverted text or generating words also leads to higher recall than does reading complete words in their normal orientation (e.g., Graf, 1982; Graf \& Ryan, 1990; Kolers, 1975; Kolers \& Ostrey, 1974; Masson \& Sala, 1978; Nairne, Reigler, \& Serra, 1991). Furthermore, versions of the compensatory processing account have also been put forth to account for these effects. These accounts propose that the impoverished condition requires additional processing during perception/identification and that this additional perceptual processing produces the mnemonic advantage (e.g., Graf, 1982; Graf \& Ryan, 1990; Hirshman, Snodgrass, Mindes, \& Feenan, 1990; Kolers, 1975; Kolers \& Ostrey, 1974; Masson \& Sala, 1978). This additional processing is usually characterized as a top-down influence in perception, as in the case of perceptual identification.

With regard to the perceptual interference effects, the top-down influences have been characterized by Hirshman et al. (1994) as semantic, lexical, or phonological in nature (recent results argue for a semantic basis of the ef- 
fect; see Mulligan, 1998). Additional research may be necessary to specify the exact nature of the underlying representations, but for the present purposes their critical attribute is that they are encoded during perception (see Hirshman et al., 1994, for evidence and discussion). As Mulligan (1996) has noted, in this sense these are representations of study items in isolation, as opposed to representations of the relations among ongoing events within the encoding context (e.g., associations between items on the list, associations between the item and spatiotemporal context, etc.). Consequently, the compensatory processing hypothesis is consistent with the item-specificrelational distinction, a framework that differentiates between encodings of items and events in isolation and encodings of relationships among items and events (see, e.g., Hunt \& Einstein, 1981; Hunt \& McDaniel, 1993; a similar distinction is that between item and associative information: e.g., Gronlund \& Ratcliff, 1989; Murdock, 1993).

The item-specific-relational framework accounts for several of the effects of perceptual interference (Mulligan, 1999) and produces additional predictions that do not naturally follow from the compensatory processing account. The central implication of this view is that perceptual interference improves recall via enhanced itemspecific encoding. A second implication is that perceptual interference does not enhance and may even impair relational processing. Several studies indicate that encoding conditions leading to greater item-specific processing often disrupt relational processing (e.g., Burns, 1996; DeLosh \& McDaniel, 1996; Nairne et al., 1991; Serra \& Nairne, 1993). ${ }^{1}$ It has been suggested that for several memory effects (such as the generation effect, bizarre image and bizarre relations effects, and the word frequency effect), the more unusual encoding condition (e.g., the generate condition, the bizarre condition, lowfrequency words) attracts greater processing of item features at the expense of relational processing (e.g., Burns, 1996; DeLosh \& McDaniel, 1996; Hunt \& McDaniel, 1993; Nairne et al., 1991; Serra \& Nairne, 1993). Casting the perceptual interference condition as the unusual encoding condition implies that enhancements in item-specific encoding may come at the cost of relational processing.

Mulligan (1999) reported results consistent with these predictions. First, Mulligan contrasted perceptual interference, a hypothesized item-specific manipulation, with list organization, a relational manipulation. Both enhanced free recall but had opposite effects on a measure of relational processing (category clustering during recall). List organization increased clustering, whereas perceptual interference decreased clustering. Second, perceptual interference reduced order memory (another type of relational information), as measured by the order reconstruction test. Third, under conditions in which order information is an important determinant of free recall, the perceptual interference effect was eliminated or reversed. In addition, although perceptual interference enhances recall and recognition memory for the occur- rence of an item, it does not enhance source memory for the encoding condition of the item (Mulligan, 1996).

The present study provides further evaluation of the item-specific account of the perceptual interference effect. This is important for several reasons. Mulligan (1999) did not provide a direct measure of the influence of itemspecific encoding on recall. The results of Mulligan are certainly consistent with the item-specific account, in showing that perceptual interference can simultaneously enhance recall while reducing relational encoding. However, these results do not provide direct, positive evidence of enhanced item-specific processing in the perceptualinterference condition. In addition, there are limitations to the use of category clustering as a measure of relational processing. First, there has been extensive debate on the extent to which category clustering provides a pure measure of relational processing (see, e.g., Burns, 1993; see Murphy, 1979, for a review). Second, the generality of clustering studies is limited because they require the use of categorized materials (see, e.g., Burns, 1993; Olofsson, 1997). Similar limitations exist for using order reconstruction as a measure of relational processing. Specifically, the order reconstruction test has been criticized as an impure measure of order memory (e.g., Neath, 1997). In addition, the typical procedure for measuring order memory (e.g., Mulligan, 1999; Nairne et al., 1991) differs from the standard study-test paradigms in a number of ways. In the order reconstruction paradigm, for example, one was multiple study-test blocks with very short (e.g., 8 item) study lists. Also, blocks alternate between requiring recall and order reconstruction tests. These procedural differences may induce strategy differences such as increased attention to serial position at encoding, or increased reliance on serial retrieval strategies during recall (see Burns, 1996, for related discussion).

Klein, Loftus, Kihlstrom, and Aseron (1989) developed an alternative method for measuring item-specific and relational influences in recall (e.g., Burns, 1993; Olofsson, 1997). In this procedure, subjects are given multiple recall tests, which sometimes leads to an overall increase in recall on later tests (a phenomenon called hypermnesia; e.g., Payne, 1987). When multiple recall tests are used, some items are recalled on later tests that have not been recalled earlier (item gains), whereas other items that have been successfully recalled earlier may not be recalled on later tests (item losses). Klein et al. (1989; Burns, 1993; McDaniel, Moore, \& Whiteman, 1998; Olofsson, 1997) demonstrated that conditions fostering item-specific encoding increase the probability of item gains, whereas conditions fostering relational encoding protect against item losses.

The item-specific-relational framework (e.g., Hunt \& McDaniel, 1993; Klein et al., 1989; McDaniel et al., 1998) suggests a theoretical account of this empirical regularity. According to this view, relational information is used during recall to guide retrieval strategies and to generate potential responses. To the extent that items (or a subset of items) are related to one another during encoding, sta- 
ble retrieval strategies should emerge and be applied consistently on successive recall trials (Hunt \& McDaniel, 1993; McDaniel et al., 1998). Thus, items associated to the consistent relational cues used at retrieval will be consistently generated, which should reduce item losses from one recall trial to the next. Item-specific encoding is hypothesized to lead to richer more extensive encoding of item attributes, leading to a more distinctive trace and facilitating discrimination among potential responses at retrieval.

Assuming that the recall of an item requires recovery of some minimal number of attributes, an item with many encoded attributes will, if not recalled on an initial trial, nevertheless have a better chance of some critical subset of those attributes being sampled on some subsequent trial (thereby producing item recovery [gains]) than would an item with fewer encoded attributes. (McDaniel et al., 1998, p. 175).

Although this theoretical speculation needs additional evaluation (e.g., McDaniel et al., 1998), the important point for the present purposes is empirical: There is a consistent positive relationship between gains and itemspecific encoding, and there is a consistent negative relationship between losses and relational encoding (Burns, 1993; Klein et al., 1989; McDaniel et al., 1998; Olofsson, 1997). Thus, to examine the components of hypermnesia is particularly useful because it (1) provides a relatively direct measure of item-specific influences on recall and (2) provides a measure of relational processing not subject to the criticisms raised by category-clustering scores or order reconstruction. Consequently, the methodology of Klein et al. provides an important converging assessment of the item-specific account of the perceptual interference effect. The item-specific account proposes that relative to the intact condition, the perceptual interference condition enhances item-specific encoding, which should lead to more item gains, and disrupts relational encoding, which should lead to more item losses.

\section{EXPERIMENT 1}

In Experiment 1, subjects were presented with a set of study words, half presented in the intact condition and half in the perceptual interference condition. Within the continuous series of study trials, the encoding conditions were presented in alternating blocks of eight trials each. After the entire study list was presented, the subjects were given two successive free recall tests. The encoding manipulation was implemented in a blocked within-subjects design (rather than a random within-subjects or between-subjects design) because it increases the probability of simultaneously observing enhancements in item-specific encoding and decrements in relational encoding (e.g., DeLosh \& McDaniel, 1996; Mulligan, 1999; Nairne et al., 1991).

\footnotetext{
Method

Subjects. Thirty-two undergraduates at Southern Methodist University participated in exchange for extra credit in psychology courses. All subjects were native English speakers.
}

Design and Materials. Encoding condition (intact vs. perceptual interference) and free recall test (1 vs. 2) were manipulated within subjects.

The study materials consisted of 48 target words of relatively high frequency (greater than 70 according to Kučera \& Francis, 1967) and four or five letters in length. The master study list was created as follows. The 48 target words were randomly ordered. The first 8 words were assigned to one of the encoding conditions (e.g., the perceptual interference condition), the next 8 to the other encoding condition (e.g., the intact condition), the next 8 to the first encoding condition (e.g., the perceptual interference condition), and so forth, so that the encoding conditions were presented in alternating sets. There were no breaks between sets; the last trial in one encoding set was immediately followed by the first trial in the next encoding set. Two versions of the master list were created, counterbalancing target words across encoding conditions. Within each study list, half of the target words were presented in the intact condition and half in the perceptual interference condition. Four additional words, similar to the target words, were used as primacy (2) and recency (2) buffers, producing study lists of 52 items. One of the primacy items was presented in the intact condition and the other in the perceptual interference condition. The same was true of the recency items.

Procedure. The subjects were tested individually. Prior to the study task, they were told that they would be presented with a series of words on a computer screen to read aloud. They were instructed to try to remember the words for a later (unspecified) memory test. They were told that some of the words would be presented very briefly and then covered with Xs (the perceptual interference condition) and that other words would be presented and left on the screen for a few seconds (the intact condition). They were also told that each word would be preceded by a prompt, consisting of a plus sign centered below the words "get ready." The participants were told that the word would appear in place of the plus sign and that they should focus their attention on the plus sign to increase their chances of identifying the words. Consistent with the instructions, each trial began with the "get ready" prompt centered on the computer screen and displayed for $.5 \mathrm{sec}$. Following the prompt, the study word was displayed in the same position as was the plus sign. In the perceptual interference condition, the study word was presented for $100 \mathrm{msec}$ and then a row of Xs was displayed in the same position for $2,400 \mathrm{msec}$. In the intact condition, the study word was presented continuously for $2.5 \mathrm{sec}$. The subjects read the words aloud while an experimenter recorded their responses.

Following the presentation of the words, the subjects completed a 3-min distractor task, a stem completion task. They were presented with a series of index cards, on each of which were the first three letters of the name of a U.S. city (e.g., "Bos__" for Boston), and they were asked to complete each word stem so that it formed a U.S. city's name.

After the distractor task, the subjects were given the first free recall memory test. They were presented with a test sheet and were asked to write down, in any order, as many words as they could recall from the study list. The test lasted $5 \mathrm{~min}$. The subjects were encouraged to use the entire time to remember as many words as possible. After the first test, the subjects were presented with another 3-min distractor task consisting of arithmetic problems on a sheet of paper. Following the arithmetic task, the second free recall test was presented. The subjects were presented with a new test sheet and were again asked to recall as many words from the study list as possible. The second test also lasted $5 \mathrm{~min}$. The subjects were again encouraged to continue trying to remember words for the entire 5 $\min$ period.

\section{Results and Discussion}

A subject must have identified at least $80 \%$ of the study words in the perceptual interference condition to be in- 
cluded in the analysis, a criterion met by all subjects in this experiment. The $\alpha$ level was .05 for all statistical tests. A sign test indicated that mean identification of study words was significantly lower in the perceptual interference condition than in the intact condition ( $98 \%$ vs. $100 \%$, respectively).

The data from the recall tests are presented in Table 1 . The number of words recalled were analyzed in a $2 \times 2$ analysis of variance, with encoding condition and recall test as within-subjects factors. The analysis revealed a significant effect of encoding condition $[F(1,31)=44.84$, $\left.M S_{\mathrm{e}}=3.61\right]$, indicating greater recall in the perceptual interference than intact condition and replicating earlier findings of a perceptual interference effect in recall (see, e.g., Mulligan, 1999). No other effects were significant $(F \mathrm{~s}<1)$. The mean numbers of intrusions were 2.59 ( $S D=$ $2.79)$ and $3.25(S D=3.79)$ for Tests 1 and 2, respectively, a difference that approached significance $(p=.085)$.

Next analyzed were the recall components. Item gains were computed as the number of words recalled on Test 2 but not on Test 1 . Item losses were computed as the number of words recalled on Test 1 but not Test 2 . The pattern of means is in accord with the item-specific account; the number of item gains and item losses were both higher in the perceptual interference than in the intact condition. However, these differences approached but did not reach the traditional significance level $(p<.09$ for both comparisons). A more general implication of the itemspecific account, which simply combines gain and loss data, was also evaluated. The item-specific account proposes that the perceptual interference condition leads to more gains (due to enhanced item-specific encoding) and more losses (due to disrupted relational encoding) than does the intact condition. Consequently, recall in this condition should be more volatile in general. Adding item gains and losses produces a measure of overall volatility. The perceptual interference condition led to significantly more recall volatility than did the intact condition [2.03, $S D=1.31$, and $1.41, S D=1.07$, respectively; $t(31)=$ 2.64]. Because the distribution of volatility scores are somewhat skewed, the data were reanalyzed with a sign test. This test also indicated significantly higher volatility for the perceptual interference condition.

Although the results of this experiment are generally in accord with the item-specific account, the specific predictions regarding gains and losses were not conclusive.
It should be noted that the overall number of gains and losses was quite low. Consequently, floor effects may have obscured differences between conditions.

Experiment 2 was designed to increase the number of item gains and losses. In addition, it should be noted that hypermnesia (i.e., increased recall on the second test) was not observed in Experiment 1. This is not especially surprising. Verbal materials typically do not produce hypermnestic recall unless the materials are presented multiple times, or are encoded under instructions that encourage elaboration or the formation of mental images (see, e.g., Belmore, 1981; Olofsson, 1997; Payne, 1986; see Payne, 1987, for a review). In Experiment 2, the study list was presented twice and subjects were encouraged to process the meaning of the study items. Payne (1986) has shown that under these conditions, hypermnesia emerges for lists of unrelated words. In addition, three successive recall tests were used because several experiments (see, e.g., Olofsson, 1997; Payne, 1986) indicate that hypermnestic effects are more pronounced with three rather than two recall tests. The combination of encoding conditions conducive to hypermnesia and an additional recall test should increase the numbers of item gains and losses, allowing for a more conclusive test of the item-specific hypothesis.

\section{EXPERIMENT 2}

\section{Method}

Subjects. Forty-nine undergraduates at Southern Methodist University participated in exchange for extra credit in psychology courses. All subjects were native English speakers.

Materials and Procedures. The materials of Experiment 2 were identical to those in Experiment 1. However, the procedures were modified on the basis of Payne's (1986) Experiment 1, which demonstrated hypermnesia with unrelated word lists. First, during the study instructions, the subjects were told that the study list would be presented twice. In addition, they were told that a good way to remember the words would be to think about the meaning of each word as it was presented. Otherwise, the study instructions were identical to those in Experiment 1. Second, the study list was presented twice, with a brief (less than 1-min) break between the two presentations. During the break, the subjects were reminded to read each word aloud and try to remember it for the later test. The second presentation was identical to the first; each word was presented in the same order and encoding condition as was the first presentation. Third, the distractor task between the study and test sessions was eliminated; the test session began immediately after the second presentation of the study list. Fourth, three 5-min recall tests were given, with no distractor tasks between recall tests. At the end of each

Table 1

Mean Number of Words Recalled and Item Gains and Losses (With Standard Deviations) as a Function of Recall Test and Encoding Condition: Experiment 1

\begin{tabular}{|c|c|c|c|c|c|c|c|c|}
\hline \multirow[b]{3}{*}{ Encoding Condition } & \multicolumn{4}{|c|}{ Test } & \multicolumn{4}{|c|}{ Recall Component } \\
\hline & \multicolumn{2}{|c|}{1} & \multicolumn{2}{|c|}{2} & \multicolumn{2}{|c|}{ Gains } & \multicolumn{2}{|c|}{ Losses } \\
\hline & $M$ & $S D$ & $M$ & $S D$ & $M$ & $S D$ & $M$ & $S D$ \\
\hline Intact & 3.53 & 1.70 & 3.75 & 2.02 & 0.81 & 0.90 & 0.59 & 0.84 \\
\hline Perceptual interference & 5.81 & 2.28 & 5.97 & 2.21 & 1.09 & 0.96 & 0.94 & 0.88 \\
\hline
\end{tabular}

Note-Number of words represents those recalled out of a possible 24 in each encoding condition. 
test, the test sheet was collected, and the instructions for the next test were immediately given.

\section{Results and Discussion}

As in Experiment 1, a subject must have identified at least $80 \%$ of the study words in the perceptual interference condition to be included in the analysis. This criterion eliminated 1 subject, rendering an effective sample size of 48 . The mean proportion of study words correctly identified was significantly lower in the perceptual interference than in the intact condition for the first presentation of the list ( $98 \%$ vs. $100 \%$, respectively), as well as the second ( $99 \%$ vs. $100 \%$, respectively).

The data from the recall tests are presented in Table 2 . The number of words recalled were analyzed in a $2 \times 3$ analysis of variance, with encoding condition and recall test as within-subjects factors. The analysis revealed two significant effects: the main effect of encoding condition $\left[F(1,47)=8.23, M S_{\mathrm{e}}=22.52\right]$, indicating greater recall in the perceptual interference than in the intact condition; and the main effect of recall test $[F(2,94)=6.76$, $\left.M S_{\mathrm{e}}=2.26\right]$, indicating that recall increased with the number of recall tests. The interaction was not significant $(F<1)$. As in Experiment 1, the perceptual interference effect was observed in recall and its size did not vary across tests. However, unlike in Experiment 1, significant hypermnesia was observed in the present experiment. Apparently, the procedural changes were effective in inducing hypermnesia (cf. Payne, 1986). The mean numbers of intrusions on Tests 1,2 , and 3 were $1.27(S D=1.34), 1.65$ $(S D=1.58)$, and $1.71(S D=1.60)$, respectively. Intrusions significantly increased over the three recall tests $\left[F(2,94)=4.12, M S_{\mathrm{e}}=2.69\right] .^{2}$

Total item gains were computed by adding item gains between Tests 1 and 2 with gains between Tests 2 and 3 (Olofsson, 1997). Total item losses were computed in the same manner. The means for total gains and losses are presented in Table 2. The results are straightforward and consistent with the item-specific account. Both item gains and item losses were significantly greater in the perceptual interference than in the intact condition $[t(47)=2.99$ and $t(47)=3.66$, respectively]. The results were unchanged when the data were analyzed with sign tests. Recall volatility was computed by summing item gains and losses. Consistent with the analysis of the recall components, the perceptual interference condition led to sig- nificantly more recall volatility than did the intact condition. The mean recall volatility was $3.63(S D=2.74)$ and $5.46(S D=3.29)$ in the intact and perceptual interference conditions, respectively $[t(47)=4.13]$. The difference was also significant by a sign test. It should be noted that the differences in results between Experiments 1 and 2 may have been due to the modified encoding conditions or the addition of Test 3 , which, by increasing the sheer number of potential item gains and losses, increased the power of Experiment 2 to detect effects (or, perhaps the differences were due to the combination of changes). The important point, however, is that the modifications introduced in Experiment 2 succeeded in raising the level of item gains and losses so that unambiguous differences between encoding conditions could be observed.

Before continuing, it is important to consider an artifactual account of present results, that perceptual interference produces more recall volatility because it leads to higher overall recall. This is unlikely for several reasons. First, as noted by Olofsson (1997), this account applies only to losses and not to gains. Item losses may be more likely for the encoding condition that produces greater recall on the first test simply because there are more items to be forgotten on the next test. However, such an account predicts fewer gains for the condition with higher recall for the complementary reason: There are fewer unrecalled items on the first test to be recalled on the second. The present results contradict this. Perceptual interference led to greater overall recall and more item gains (there are several other reports of a similar pattern of higher recall associated with more gains: e.g., Belmore, 1981; Burns, 1993). Second, contrary to this artifactual account, there are several studies in which the condition with higher initial recall has produced fewer, rather than more, item losses (e.g., Burns, 1993; Klein et al., 1989, Payne, 1986). Not surprisingly, this typically occurs when encoding conditions encourage relational processing (e.g., Burns, 1993; Klein et al., 1989). More generally, Payne's (1986) results indicate little relationship between the components of hypermnesia (i.e., gains and losses) and initial level of recall. Third, an additional analysis indicated that perceptual interference led to relatively as well as absolutely more items losses. For each subject, the proportions of item losses between Tests 1 and 2 and between Tests 2 and 3 were computed relative to the number of items recalled on the first test in each pair. The

Table 2

Mean Number of Words Recalled and Item Gains and Losses

(With Standard Deviations) as a Function of

Recall Test and Encoding Condition: Experiment 2

\begin{tabular}{|c|c|c|c|c|c|c|c|c|c|c|}
\hline \multirow[b]{3}{*}{ Encoding Condition } & \multicolumn{6}{|c|}{ Test } & \multicolumn{4}{|c|}{ Recall Component } \\
\hline & \multicolumn{2}{|c|}{1} & \multicolumn{2}{|c|}{2} & \multicolumn{2}{|c|}{3} & \multicolumn{2}{|c|}{ Gains } & \multicolumn{2}{|c|}{ Losses } \\
\hline & $M$ & $S D$ & $M$ & $S D$ & $M$ & $S D$ & $M$ & $S D$ & $M$ & $S D$ \\
\hline Intact & 7.75 & 3.99 & 8.04 & 4.25 & 8.50 & 4.41 & 2.19 & 2.10 & 1.44 & 1.40 \\
\hline Perceptual interference & 9.40 & 3.79 & 9.52 & 3.89 & 10.19 & 3.87 & 3.15 & 2.15 & 2.31 & 1.76 \\
\hline
\end{tabular}

Note-Number of words represents those recalled out of a possible 24 in each encoding condition. 
average of these two proportions is the relative loss score. The mean relative loss score was $.09(S D=.08)$ and .13 $(S D=.11)$ in the intact and perceptual interference conditions, respectively. This difference was significant by $t$ test $[t(47)=1.98, p=.053]$ and by sign test $(p<.05)$. See Burns (1993), Klein et al. (1989), and Olofsson (1997) for additional evidence against this artifactual account.

\section{GENERAL DISCUSSION}

The hypothesis investigated in the present study is that perceptual interference enhances item-specific processing and disrupts relational processing. Consistent with this account, perceptual interference increased the number of item gains across multiple recall tests, implying enhanced item-specific encoding, and increased the number of losses, implying disrupted relational processing (Klein et al., 1989). This interpretation is consistent with the conditions set forth by Burns (1993, pp. 171-172), who argued that the item gain and loss measures are valid when the encoding condition with more gains and losses also leads to greater overall recall (see also Olofsson, 1997). This is the exact pattern of results in the present study: In comparison with the intact condition, the perceptual interference condition led to more gains and losses coupled with greater overall recall.

The present results provide converging evidence for the item-specific hypothesis and fit well with earlier results. As noted in the introduction, Mulligan (1999) reported that perceptual interference reduced relational processing as measured by category clustering and serial order reconstruction. The results of the item losses converge with these results. In addition, the item gains provide independent and relatively direct evidence of enhanced item-specific encoding.

Several additional results are accommodated by the item-specific account. First, perceptual interference dissociates performance on the matched implicit and explicit tests of category exemplar production and category cued recall, enhancing recall but leaving conceptual priming unaffected (Mulligan, 1996). In category cued recall, subjects attempt to produce exemplars that are from a given category and that have appeared on the study list; in category exemplar production, the task is simply to produce exemplars from given categories. Because category cued recall requires finer discriminations than does category exemplar production, we might expect that category cued recall has a greater reliance on item-specific encoding (Weldon \& Coyote, 1996, came to a similar conclusion; see Mulligan, Guyer, \& Beland, 1999, for additional evidence). Thus, the item-specific-relational distinction provides an account of the dissociative effects of perceptual interference on these two tasks (Mulligan, 1996).

Second, the perceptual interference effect is more readily demonstrated on recognition than on free recall memory tests (Hirshman \& Mulligan, 1991; Nairne, 1988). Recognition is more sensitive than free recall to item-specific information (see, e.g., Hunt \& Einstein,
1981; Hunt \& McDaniel, 1993). Accordingly, the effects of perceptual interference on recognition are expected to be more robust than the effects of perceptual interference on recall. Third, perceptual interference does not enhance memory in between-subjects designs (Mulligan, 1999; Westerman \& Greene, 1997). Other effects attributed to enhanced item-specific processing, such as orthographic distinctiveness, the generation effect, and the bizarre-imagery effect, are also more likely to be found in within-subjects (or mixed-list) designs than in betweensubjects (or pure list) designs (e.g., Hunt \& Elliot, 1980; McDaniel \& Einstein, 1986; Serra \& Nairne, 1993; see Hunt \& McDaniel, 1993, for a review). According to the item-specific-relational framework, item-specific processing creates distinctive memory traces. The effects of item-specific encoding are diminished or eliminated when encoding conditions are implemented in pure study lists, because the consistency of encoding conditions renders the favored encoding condition less distinctive (see, e.g., Hunt \& McDaniel, 1993; McDaniel \& Einstein, 1986).

Finally, as has been noted in the introduction, other encoding manipulations such as the generate manipulation may have effects that are similar to those of perceptual interference. In particular, Westerman and Greene (1997) have documented several similarities between the effects of perceptual interference and generation. In comparison with reading intact words at study, generating a word from a cue or identifying a word in the perceptual interference condition typically leads to superior memory on explicit tests, such as free and cued recall and recognition (Westerman \& Greene, 1997). Likewise, both the generation effect and the perceptual interference effect are less likely to occur with nonword stimuli and in betweensubjects designs. In addition, both generation and perceptual interference improve item memory but typically disrupt order memory (Mulligan, 1996; Nairne et al., 1991; Serra \& Nairne, 1993).

These interesting similarities are generally consistent with the item-specific-relational account and suggest that the generation effect should produce a pattern of hypermnestic results similar to those found with perceptual interference. Specifically, relative to a reading condition, generating at encoding should produce more item gains as well as more item losses across multiple recall trials. Little research on the relationship between generation and hypermnesia has been conducted. To my knowledge, only one study (Payne \& Wenger, 1992) has reported the recall components needed to evaluate this relationship. In this study, subjects either produced the answers to relatively easy riddles (the generate condition) or read the single-word answers in isolation (a third, picture study condition is not relevant for the present purposes). In comparison with the read condition, the generate condition led to significantly greater recall volatility and greater item gains and losses. Thus, generation (at least as instantiated by Payne \& Wenger, 1992) and perceptual interference produce very similar results in the recall components of hypermnesia. 
The similarities between the generation and perceptual interference effects are intriguing and suggest that the representational bases of these effects may overlap (Westerman \& Greene, 1997). However, enthusiasm for the similarity of these effects must be tempered by the observation that generation and perceptual interference have different effects on implicit memory tests. First, generation but not perceptual interference enhances priming in the category exemplar production, a conceptual implicit test of memory (Mulligan, 1996; Srinivas \& Roediger, 1990). In addition, perceptual priming tasks typically exhibit a reversed generation effect in which the read condition leads to greater priming than does the generate condition (see Roediger \& McDermott, 1993, for a review). However, perceptual identification and speeded naming (both perceptual implicit tests) show no difference between the intact and perceptual interference conditions (Hirshman \& Mulligan, 1991; Hirshman et al., 1994). Additional research will be necessary to determine the extent of the similarity between these two mnemonic effects and whether the similarities are limited to explicit memory.

\section{REFERENCES}

Balota, D. A. (1990). The role of meaning in word recognition. In D. A. Balota, G. B. Flores d'Arcais, \& K. Rayner (Eds.), Comprehension processes in reading (pp. 9-32). Hillsdale, NJ: Erlbaum. BELMORE, S. M. (1981). Imagery and semantic elaboration in hypermnesia for words. Journal of Experimental Psychology: Human Learning \& Memory, 7, 191-203.

BurNs, D. J. (1993). Item gains and losses during hypermnesic recall: Implications for the item-specific-relational information distinction. Journal of Experimental Psychology: Learning, Memory, \& Cognition, 19, 163-173.

BuRNS, D. J. (1996). The item-order distinction and the generation effect: The importance of order information in long-term memory. American Journal of Psychology, 109, 567-580.

CARR, T. H., \& Dagenbach, D. (1990). Semantic priming and repetition priming from masked words: Evidence for a center-surround attentional mechanism in perceptual recognition. Journal of Experimental Psychology: Learning, Memory, \& Cognition, 16, 341-350.

DeLosh, E. L., \& MCDANiEl, M. A. (1996). The role of order information in free recall: Application to the word-frequency effect. Journal of Experimental Psychology: Learning, Memory, \& Cognition, 22, 1136-1146.

Graf, P. (1982). The memorial consequences of generation and transformation. Journal of Verbal Learning \& Verbal Behavior, 21, 539-548.

Graf, P., \& Ryan, L. (1990). Transfer-appropriate processing for implicit and explicit memory. Journal of Experimental Psychology: Learning, Memory, \& Cognition, 16, 978-992.

GRonlund, S. D., \& RaTCLIFF, R. (1989). Time course of item and associative information: Implications for global memory models. Journal of Experimental Psychology: Learning, Memory, \& Cognition, $15,846-858$.

Hirshman, E., \& Mulligan, N. W. (1991). Perceptual interference improves explicit memory but does not enhance data-driven processing. Journal of Experimental Psychology: Learning, Memory, \& Cognition, 17, 507-513.

Hirshman, E., Snodgrass, J. G., Mindes, J., \& Feenan, K. (1990). Conceptual priming in fragment completion. Journal of Experimental Psychology: Learning, Memory, \& Cognition, 16, 634-647.

Hirshman, E., Trembath, D., \& Mulligan, N. W. (1994). Theoretical implications of the mnemonic benefits of perceptual interference. Journal of Experimental Psychology: Learning, Memory, \& Cognition, 20, 608-620.
HUNT, R. R., \& EinStein, G. O. (1981). Relational and item-specific information in memory. Journal of Verbal Learning \& Verbal Behavior, 19, 497-514.

HunT, R. R., \& Elliot, J. M. (1980). The role of nonsemantic information in memory: Orthographic distictiveness effects on retention. Journal of Experimental Psychology: General, 109, 49-74.

HUnT, R. R., \& MCDANIEL, M. A. (1993). The enigma of organization and distinctiveness. Journal of Memory \& Language, 32, 421-445.

Klein, S. B., Loftus, J., Kinlstrom, J. F., \& Aseron, R. (1989). Effects of item specific and relational information on hypermnesic recall. Journal of Experimental Psychology: Learning, Memory, \& Cognition, 15, 1192-1197.

KOLERS, P. A. (1975). Memorial consequences of automatized encoding. Journal of Experimental Psychology: Human Learning \& Memory, 1, 689-701.

Kolers, P. A., \& OSTREY, D. J. (1974). Time course of loss of information regarding pattern analyzing operations. Journal of Verbal Learning \& Verbal Behavior, 13, 599-612.

KUČERA, H., \& FRANCIS, W. N. (1967). Computational analysis of presentday American English. Providence, RI: Brown University Press.

Masson, M. E. J., \& SALA, L. (1978). Interactive processes in sentence comprehension and reading. Cognitive Psychology, 10, 244-270.

MCDANIEL, M. A., \& EinSTEIN, G. O. (1986). Bizarre imagery as an effective memory aid: The importance of distinctiveness. Journal of Experimental Psychology: Learning, Memory, \& Cognition, 12, 54-65.

McDaniel, M. A., Moore, B. A., \& Whiteman, H. L. (1998). Dynamic changes in hypermnesia across early and late tests: A relational/ item-specific account. Journal of Experimental Psychology: Learning, Memory, \& Cognition, 24, 173-185.

Mulligan, N. W. (1996). The effects of perceptual interference at encoding on implicit memory, explicit memory, and memory for source. Journal of Experimental Psychology: Learning, Memory, \& Cognition, 22, 1067-1087.

Mulligan, N. W. (1998). Perceptual interference at encoding enhances recall for high- but not low-imageability words. Psychonomic Bulletin \& Review, 5, 464-469.

Mulligan, N. W. (1999). The effects of perceptual interference at encoding on organization and order: Investigating the roles of itemspecific and relational information. Journal of Experimental Psychology: Learning, Memory, \& Cognition, 25, 54-69.

Mulligan, N. W., Guyer, P. S., \& Beland, A. (1999). The effects of levels-of-processing and organization on conceptual implicit memory in the category exemplar production test. Memory \& Cognition, 27, 633-647.

MURDOCK, B. B. (1993). TODAM2: A model for the storage and retrieval of item, associative, and serial-order information. Psychological Review, 100, 183-203.

MuRPHY, M. D. (1979). Measurement of category clustering in free recall. In C. R. Puff (Ed.), Memory organization and structure (pp. 5183). New York: Academic Press.

NAIRNE, J. S. (1988). The mnemonic value of perceptual identification. Journal of Experimental Psychology: Learning, Memory, \& Cognition, 14, 244-255.

Nairne, J. S., Reigler, G. L., \& Serra, M. (1991). Dissociative effects of generation on item and order retention. Journal of Experimental Psychology: Learning, Memory, \& Cognition, 17, 702-709.

NeATh, I. (1997). Modality, concreteness, and set-size effects in a free reconstruction of order task. Memory \& Cognition, 25, 256-263.

OlofsSON, U. (1997). Win some, lose some: Hypermnesia for actions reflects increased item-specific processing. Memory \& Cognition, 25, 797-800.

PAYNE, D. G. (1986). Hypermnesia for pictures and words: Testing the recall level hypothesis. Journal of Experimental Psychology: Learning, Memory, \& Cognition, 12, 16-29.

PAYNE, D. G. (1987). Hypermnesia and reminiscence in recall: A historical and empirical review. Psychological Bulletin, 101, 5-27.

PAYNe, D. G., \& Wenger, M. J. (1992). Repeated recall of pictures, words, and riddles: Increasing subjective organization is not sufficient for producing hypermnesia. Bulletin of the Psychonomic Society, 30, 407-410. 
Plaut, D. C., McClelland, J. L., Seidenberg, M. S., \& Patterson, K. (1996). Understanding normal and impaired word reading: Computational principles in quasi-regular domains. Psychological Review, 103, 56-115.

ROEDIGER, H. L., III, \& MCDERMOTT, K. B. (1993). Implicit memory in normal human subjects. In F. Boller \& J. Grafman (Eds.), Handbook of neuropsychology (Vol. 8, pp. 63-131). Amsterdam: Elsevier.

SeIDENBERG, M. S., \& MCClelland, J. L. (1989). A distributed developmental model of word recognition and naming. Psychological Review, 96, 523-568.

SerRa, M., \& NaIRNE, J. S. (1993). Design controversies and the generation effect: Support for an item-order hypothesis. Memory \& Cognition, 21, 34-40.

SRINIVAS, K., \& Roediger, H. L., III (1990). Classifying implicit memory tests: Category association and anagram solution. Journal of Memory \& Language, 29, 389-412.

Strain, E., Patterson, K., \& Seidenberg, M. S. (1995). Semantic effects in single-word naming. Journal of Experimental Psychology: Learning, Memory, \& Cognition, 21, 1140-1154.

WELDON, M. S., \& CoYOTE, K. C. (1996). Failure to find the picture superiority effect in implicit conceptual memory tests. Journal of Experimental Psychology: Learning, Memory, \& Cognition, 22, 670-686.
Westerman, D. L., \& Greene, R. L. (1997). The effects of visual masking on recognition: Similarities to the generation effect. Journal of Memory \& Language, 37, 584-596.

\section{NOTES}

1. This research has predominantly focused on order information, which is typically conceived of as one type of relational information (e.g., Burns, 1996; DeLosh \& McDaniel, 1996; Hunt \& McDaniel, 1993).

2 . The intrusion data raise the question of whether hypermnesia reflects increased accuracy in recall or is an artifact of increased guessing (note that the effects of perceptual interference could not be undermined by differences in guessing across tests). To investigate this, corrected recall scores were computed for each test by summing the number of words recalled in the perceptual interference and intact conditions, and subtracting the number of intrusions. Corrected recall scores significantly increased across recall tests $\left[F(2,94)=3.40, M S_{\mathrm{e}}=5.53\right]$, indicating that the observed hypermnesia was not an artifact of guessing.

(Manuscript received July 27, 1998; revision accepted for publication July 23,1999 .) 